\title{
The progress of post-treatment restricted mouth opening in oral and maxillofacial malignant tumor patients
}

\author{
Hao Wu ${ }^{1}$, Zijie Zhou ${ }^{2}$, Chengyao Zhang ${ }^{2}$, Shukun Shen ${ }^{2}$, Jiannan Liu ${ }^{2,3,4}$, Chenping Zhang ${ }^{2,3,4}$ \\ ${ }^{1}$ Weifang Medical University, College of Stomatology, Weifang, China; ${ }^{2}$ Department of Oral Maxillofacial-head and Neck Oncology, Shanghai \\ Ninth People's Hospital, College of Stomatology, Shanghai Jiao Tong University School of Medicine, Shanghai, China; ${ }^{3}$ National Clinical Research \\ Center for Oral Diseases, Shanghai, China; ${ }^{4}$ Shanghai Key Laboratory of Stomatology \& Shanghai Research Institute of Stomatology, Shanghai, \\ China \\ Contributions: (I) Conception and design: All authors; (II) Administrative support: Corresponding authors; (III) Provision of study materials or \\ patients: All authors; (IV) Collection and assembly of data: All authors; (V) Data analysis and interpretation: All authors; (VI) Manuscript writing: All \\ authors; (VII) Final approval of manuscript: All authors. \\ Correspondence to: Jiannan Liu; Chenping Zhang. Department of Oral and Maxillofacial-Head \& Neck Oncology, Ninth People's Hospital Affiliated \\ to Shanghai Jiao Tong University School of Medicine, No. 639, Zhi Zao Ju Road, Shanghai 200011, China. \\ Email: laurence_lin@163.com; zhang.chenping@hotmail.com.
}

\begin{abstract}
Oral and maxillofacial malignant tumor seriously threaten the physical and mental health of patients, especially advanced malignant tumor requiring a comprehensive sequence of surgery-based treatment, resulting in facial deformity, difficulties with chewing, dysphagia and asophia. Among them, trismus (restricted mouth opening) is one of the most common complications after treatment of oral and maxillofacial malignant tumor. In severe cases, they may even suffer from trismus and eating difficulties, finally leading to malnutrition and even cachexia. Therefore, it not only affects the quality of life of the patients and even endangers their lives, but also brings heavy social and economic burden. How to effectively prevent and treat posttreatment trismus is a clinical problem which is easy to be ignored by oral and maxillofacial surgeons and needs to be solved urgently. In recent years, restricted mouth opening (trismus) after post-treatment of oropharyngeal, nasopharyngeal and other head and neck malignant tumors have been widely studied, but restricted mouth opening after comprehensive treatment of oral cancer has been neglected. We therefore conducted a systematic review with 3 aims: (I) to clear the definition of restricted mouth opening and trismus; (II) to identify epidemiology and pathogenesis of restricted mouth opening or trismus; and (III) to summarize the methods of interventions or treatment of oral and maxillofacial malignant tumor patients with restricted mouth opening or trismus.
\end{abstract}

Keywords: Oral and maxillofacial malignant tumor; comprehensive treatment; restricted mouth opening; trismus

Received: 09 August 2020; Accepted: 25 January 2021; Published: 30 March 2021.

doi: 10.21037/fomm-20-52

View this article at: http://dx.doi.org/10.21037/fomm-20-52

\section{Introduction}

Oral and maxillofacial malignant tumor is accounting for about $7 \%$ of systemic malignancies, becoming a global health concern (1). Patients with oral and maxillofacial malignant tumor may have a variety of complications after surgery and/or radiotherapy/chemotherapy, including dysphagia dysfunction, limited mouth opening or trismus (2). Restricted mouth opening (RMO) or trismus, which can develop as a result of the disease or its treatment, and radiotherapy has been reported as one of the most common causes (3). RMO or trismus in patients with oral and maxillofacial malignant tumor may lead to limited daily activities such as eating, chewing, swallowing, breathing, and speaking.

Restricted mouth opening or trismus also can lead to other problems such as severe pain, weight loss, 
psychological difficulties (including low self-esteem, depression, and suicidal tendencies), poor oral hygiene, negative influence on quality of life (QoL), and interferes with tumor surveillance in the postoperative period (4-6). Treatment of RMO or trismus can be used by conservative therapy (medication or physical therapy), surgery or rehabilitation therapy (exercise therapy). It has been confirmed that (7), exercise therapy is the main method to effectively prevent the occurrence of limitation in mouth opening and reduce its severity. This article reviews the occurrence and prevention of RMO or trismus in patients with oral and maxillofacial malignant tumor, in order to provide clinical reference for promoting the implementation of mouth opening training.

\section{The definition, epidemiology and pathogenesis of RMO or trismus}

\section{The definition}

Restricted mouth opening and trismus are common terms used in oral oncology, which is difficulty in mouth opening. RMO as reported in the literature is a clinical symptom in which the mouth opening is restricted owing to any cause (8). Historically, the term trismus was used to refer to patients who were unable to open their mouth due to tetanus (9). Currently, trismus is used to indicate severely RMO of any aetiology. Among the various terms used in literatures, trismus in oral cancer is defined as a tonic contraction of the muscles of mastication due to any abnormal condition or disease, with maximum mouth opening (MMO) of $\leq 35 \mathrm{~mm}$ (either the interincisal distance or the distance between the upper and lower alveolus) (4). It may be a result of tumor involving masticatory muscles, infection, surgery or radiation. However, a literature emphasizes that trismus mainly indicates the restricted mouth opening due to dysfunction of the muscles of mastication and should not be used in all instances where there is limitation of mouth opening, which is suitable for restricted mouth opening caused by reasons other than muscle dysfunction (8).

According to the literature, classification of trismus based on clinical examination of the maximal interincisal distance (MID), which shows the vertical dimension from maxillary incisor margin to mandibular incisor margin in maximum opening position (8). In edentulous patients, it is measured the distance between the maxillary and mandibular alveolar ridges (10). Standard of restriction of the mouth opening with reference to late adverse reactions of normal tissues (late effects of normal tissues/subjective and objective management analysis, LENT/SOMA), trismus is classified into four grades as follows: grade 1, limited mouth opening, MID from 21 to $30 \mathrm{~mm}$; grade II, difficulty in feeding, MID from 11 to $20 \mathrm{~mm}$; grade III, difficulty in feeding soft food, MID from 5 to $10 \mathrm{~mm}$; and grade IV, nasal feeding, MID $<5 \mathrm{~mm}$ (11). Grades III and IV are defined as severe trismus $(11,12)$.

\section{The prevalence}

The reported prevalence of limitation in mouth opening in patients with oral-maxillofacial malignant tumors ranges from $8 \%$ to $62 \%$ (13). The patients were a majority male, with an average age from 53.5 to 65.9 years old $(14,15)$. It may be related to the tumor clinical stage, tumor site, treatment(s) used, radiotherapy site, radiotherapy dose, radiotherapy type, follow-up time after treatment and sample variation. The incidence of restriction in mouth opening before and after operation is quite different (16). Patients with oral and maxillofacial malignant tumor, especially carcinoma of the buccal mucosa or oropharyngeal cancer, have preoperative trismus. Studies have shown that $55.6 \%$ of patients with oral cancer had trismus before surgery, and $80.2 \%$ of patients with buccal mucosa cancer had trismus after surgery (17). Another study of oral cancer found that $47 \%$ of patients had preoperative trismus, $71 \%$ of patients had postoperative trismus, and the prevalence of trismus at 6 months after surgery or radiotherapy can reach $79 \%$ (3). Another study of oral and oropharyngeal cancer study found that $30 \%$ of patients have preoperative trismus, $65 \%$ of patients have postoperative trismus (18).

The location or size of the primary lesion had a significant effect on limitation in mouth opening. The degree of opening in patients with $\mathrm{T} 3-4$ stage was significantly less than that in patients with T1-2 stage (24 and $35 \mathrm{~mm}$, respectively) (19,20), indicating that larger tumors is associated with a higher risk of developing trismus. As far as tumor location is concerned, patients with posterior buccal mucosal carcinoma (attachment of masticatory muscles) have the highest incidence of mouth opening limitation (11), patients with oropharyngeal cancer also have a higher risk (5). Fifty-five percent of patients with parapharyngeal malignancies have restricted mouth opening symptoms at the time of diagnosis (21), while patients with tumors located in the posterior molar area or oropharynx are at higher risk of restriction of mouth opening than those 
located at the floor of mouth or anterior $2 / 3$ tongue.

In terms of the treatments of different types of head and neck tumor, trismus has been reported to $5-32 \%$ of the patients treated for maxillary sinus carcinoma (16,22-24), $0-69 \%$ of patients treated for oral and oropharyngeal cancer $(25-27)$ and $0-36 \%$ of patients treated for nasopharyngeal cancer (28-30). Based on meta-analysis (14), the prevalence of trismus in patients with head and neck carcinoma increased from $17.3 \%$ at baseline to a peak of $44.1 \%$ at 6 months, decreased to $32.1 \%$ at 12 months, up to $36.1 \%$ at 24 months and continued in average $32.6 \%$ at $3-10$ years.

Effect of different treatments and the follow-up time after treatment on limitation in mouth opening are different. van der Geer et al. (31) reported that MMO of patients treated by surgery alone was $37 \mathrm{~mm}$ and treated by radiotherapy alone $33 \mathrm{~mm}$. Previous literature has indicated that the use of radiotherapy decreased significantly patient's mouth opening, up to $38 \mathrm{~mm}$ for patients without radiotherapy and $27 \mathrm{~mm}$ for adjuvant radiotherapy $(32,33)$. After radiotherapy, MMO of patients decreased gradually (34). On average, MMO decreased at a rate of $1.3 \%$ per month during radiotherapy (first 9 weeks). In the first 9 months after radiotherapy, MMO decreased by $2.4 \%$ per month. Between 12 and 24 months after radiotherapy, MMO decreased by $0.2 \%$ per month (31).

This risk increases in proportion to radiation dose and irradiated volume of anatomical structures involved $(13,35,36)$. Since most patients have a dose of $60-65$ Gy, it is not possible to determine a critical dose for radiotherapy. However, previous studies have shown that radiation doses greater than 70 Gy lead to worse trismus $(2,28)$, and another literature reported that the doses of radiotherapy exceeding of $60 \mathrm{~Gy}$ are more likely to lead to trismus (20). The use of soft tissue or composite free flap in the reconstruction of head and neck cancer patients will also reduce mouth opening but this group of patients tended to be more severe and more likely to undergo adjuvant radiotherapy (37).

Studies have shown that there is a significant correlation between the absorbed dose to the chewing structures and mouth opening. As doses of radiotherapy increase, the prevalence of the trismus increases. It was reported that for every $10 \mathrm{~Gy}$ of additional radiation added to the medial pterygoid muscles, the probability of developing trismus increased by $24 \%$ (38). A systematic review of trismus caused by cancer treatment showed that the prevalence of patients with conventional radiotherapy was $25.4 \%$, while that of patients with intensity modulated radiotherapy (IMRT) was 5\% (35). Furthermore, the dosimetric studies have shown that IMRT reduces the occurrence of trismus through this treatment modality (39).

\section{Etiopathogenesis and causative mechanisms of RMO}

Restricted mouth opening or trismus can be caused by either intra-articular or extra-articular problems (40). The pathogenesis of RMO or trismus in patients with oral-maxillofacial malignant tumor can be summarized as follows:

(I) Tumor factors: RMO or trismus can be caused by local invasion of the primary or metastatic tumor to chewing structures (2) [including the masseter, medial pterygoid muscles, lateral pterygoid muscles, their neural innervation, the temporomandibular joint (TMJ) and/or other supportive tissues], such as infratemporal fossa tumor, pterygopalatine concave tumor, maxillary sinus malignant tumor invading posterior wall, oral cancer, oropharyngeal cancer, etc. In addition, chewing betel nut can cause oral submucous fibrosis (OSMF), and then lead to restricted mouth opening (41).

(II) Surgical factors: Patients with oral cancer (especially in advanced stage) need extensive intraoral tissue removal and reconstruction (skin graft, local adjacent flap transfer or free flap). Surgery in the area of TMJ area or the masticatory muscles area may result in RMO. Postoperative tissue adhesion, split thickness skin graft, surgical scar formation may reduce the mouth opening due to contraction and fibrosis (42). Inadequate tissue repair, inflammation, long-term fistula after surgery will also lead to limitation of mouth opening. Additionally, RMO may be the result of a mandibular fracture because the material used to reconstruct the mandible failed (40).

(III) Radiotherapy factor: Fibrosis is thought to be one of the biological mechanisms that lead to late radiation-induced side effects $(6,34,43)$. When irradiating TMJ and masticatory muscles, patients have the risk of developing trismus $(44,45)$. Tumor locations associated with this type of radiotherapy include nasopharynx, oral cavity, base of tongue, oropharynx, salivary gland, maxilla or mandible. Radiotherapy not only causes fibrosis of the masticatory muscles, but also causes fibrosis of the soft tissues and salivary glands included in the irradiation fields. Reduced the lubrication of 
oral mucosal and muscle dysfunction caused by radiation-induced reduction in saliva secretion may cause oral pain and stimulated the development of trismus (46).

Studies have shown that immediate fixation of fixed joints begins to produce degenerative changes, including synovial fluid thickening and cartilage thinning (35). The directly effect of radiation on muscle eventually leads to fibrosis and contracture, which gradually develops about 9 weeks after the completion of the treatment $(34,47)$. In addition, radiation-induced cellulitis of the head and face and periodontitis in the molar area can also lead to severe mouth opening difficulties. Radiationinduced oral mucositis and dermatitis also occur during radiotherapy positively correlated with the degree of difficulty in opening mouth (48).

(IV) Psychological factors: Due to the psychological fear of pain (malignant tumor pain, postoperative wound pain, roentgen ulcer, oral mucositis caused by local pain), patients may be reduced the range of motion of the mandible. Postoperative pain in the oral cavity may restrict patient's movement and aggravate restricted opening due to lack of activity (49). As the jaw movement is reduced, the muscle will show signs of atrophy in 3 days. Similarly, fixed joint begins to show degenerative changes, such as synovial fluid thickening and cartilage thinning (35).

\section{Present situation of prevention and treatment of RMO or trismus}

\section{Drugs for the treatment of trismus.}

Some studies have found that the use of pentoxifylline in patients with fibrosis caused by radiotherapy can significantly increase the range of mandibular motion. The effect of pentoxifylline on cytokine-mediated inflammatory response. Therefore, the role of cytokines in the radiation response process of tumors and normal tissues provides further opportunities for the combination of drugs and radiation. And there was a literature said that pentoxifylline, a methylxanthine derivative, has a broad application prospects in the field of cancer (50). However, some literatures reported that drug treatments may be ineffective, such as pentoxifylline and hyperbaric oxygen $(50,51)$. Although injecting botulinum toxin into the masticatory muscles can effectively relieve pain, it has been reported to have potential benefits in the treatment of certain complications of radiation fibrosis syndrome $(20,52)$.

\section{Surgical treatment of $R M O$}

The common surgeries to improve limited mouth opening include reconstruction of tissues of traditional nonmicrosurgical surgery (53-55) (buccal fat pads, tongue flaps, nasolabial flap, split thickness skin graft, myotomy and coronoidectomy), microsurgical free flap reconstruction surgery (56), the jaw joints endoscopic release, the joint cavity lavage under endoscopic technique.

Studies have shown that after surgical treatment (complete release or removal of fibrotic tissue, marginal mandibulectomy, ipsilateral resection of the coronoid process, and ipsilateral myotomy of the temporalis muscle's insertion, medial pterygoid muscle, and masseter muscle), sufficient free tissue flap can be used to repair the defect, and postoperative rehabilitation training can effectively alleviate the prevalence of limitation in mouth opening (57).

Due to radiation-induced masticatory muscles fibrosis attached to the coracoid process, coronoidectomy can prevent and cure trismus following radiotherapy. In surgically treated patients, when the tumor approaches the coronoid process, coronoidectomy is recommended to prevent the progression of RMO during tumor resection (20). After coronal resection, the patient's MID usually increases immediately by at least $20 \mathrm{~mm}$, and half improves by $30 \mathrm{~mm}$.

After tumor excision, simultaneous reconstruction using an anterior thigh flap, MMO immediately increased at 21.4 and $16.5 \mathrm{~mm}$ after 1 year (58). Although a few studies have found surgical treatment improves outcome, there are no guidelines as to when the surgery must be initiated in such situations.

\section{Radiotherapy treatment}

Radiotherapy is one of the most important causes of trismus in patients with oral and maxillofacial malignant tumors. Studies showed that in up to $50 \%$ of patients with radiotherapy suffer from temporomandibular joint and masticatory muscle injury(5), which results in reactive exudation and adhesion, and then fibrosis forms contracture, with limited opening and even closed teeth. the maximum opening degree after radiotherapy is $18-32 \%$ less than that before radiotherapy (33).

Since 1989 (59), radiation techniques such as threedimensional conformal radiotherapy (3DCRT) and 
intensity-modulated radiotherapy (IMRT) have been used to treat head and neck cancer and reduce radiation damage to normal tissues.

Graff et al. (60) showed that IMRT produces less oral complications than conventional radiotherapy. Radiation induces masticatory muscles fibrosis, leading to loss of function and necrosis of bone and soft tissue (34). Based on the above information, the best way to prevent radiationinduced RMO is to minimize the radiation doses to the TMJ and the muscles of mastication. Nowadays, the widespread use of new forms of RT, and especially IMRT, not only the accuracy of radiotherapy and protects normal tissues, but also has a lower risk of osteonecrosis of the jaw, less oral dryness, relatively mild pain, and a lower incidence of oral mucosal inflammation that greatly improves patient quality of life without compromising the necessary therapeutic dose (3).

\section{Physical therapy and exercise therapy}

Exercise therapy is the main treatment of trismus, although there is a limited evidence to prove the effectiveness of exercise therapy. As the reported that trismus evolved most rapidly during the first 9 months after therapy and then ceased to progress in most patients (61). Oral opening training should start as soon as possible after surgery and should begin during RT. Exercise should be carried out as frequently as possible, especially in the vertical and horizontal range of exercise practice.

Various treatments for RMO (trismus) have been reported, including use of supportive measures, such as frequent use of muscle relaxants muscle relaxants, hot compresses or hyperbaric oxygen (46) and range of motion exercises, use of mechanically assisted like rubber stoppers, wooden tongue blades, spatulas, finger stretching, spring appliances, the jaw stretching device, and the TheraBite exercise rehabilitation system.

According to literature report that the exercise program includes the following three steps: (I) warm-up exercise, that is, opening the jaw 10 times without using the jaw device, and make the jaw perform 10 times small sideway movements; (II) passive stretching with the jaw-mobilizing device and conventional mouth devices (tongue-spatula, etc.) for $30 \mathrm{~s}$ (if possible), to be repeated five times; and (III) five repetitions of active exercise (bite toward resistance). Asking patients to relax in each session and to gradually increase the amount and intensity of the exercise to avoid pain or injury. The protocol is a 12 -week structured exercise plan that includes exercise 9 times per day. During the program, the patients were evaluated by oral surgeons, who measured MIO after 12 weeks of intervention (62).

The cheapest option is to use a tongue depressor, which has been used to mobilize the jaw for many years, but a search of the literature has not found any studies that proved an obvious improvement in the treatment of trismus with tongue depressor (35). The current clinical evidence shows that the maximum mouth opening of patients with exercise intervention or using TheraBite $^{\circledR}$ exercise rehabilitation system is significantly better than that of patients without auxiliary exercise $(7,63)$.

For patients who exercise with TheraBite ${ }^{\circledR}$ exercise rehabilitation system, they will be asked to follow two protocol: (I) the 5-5-30 protocol for 5 sessions per day, five openings/closing per session, $30 \mathrm{~s}$ stretch for each opening; (II) the 7-7-7 protocol which is seven sessions per day, seven openings/closing per session, seven seconds stretch for each opening. This system has been selected after consultation with Platon medical which manufactures the equipment and has global experience in using the device. About $3 / 4$ weeks after surgery, the patient will start using TheraBite ${ }^{\circledR}$ to exercise. Postsurgical maximum mouth opening and health-related quality of life (HRQoL) assessments will be performed at 3 to 4 weeks after surgery. This is the optimal time before the start of radiation therapy. Follow-up evaluations will take place at 3 and 6 months after the start of the intervention (64). According to the literature, it is essential that the use of TheraBite ${ }^{\circledR}$ should be encouraged and maintained maximum benefits for patients with mandibular hypomobility as early as possible (65).

Before starting the intervention, the medical staff asked the patients to fill in a questionnaire to assess the patient's open-mouth condition and specify a personalized intervention protocol, so as to improve the obedience of patient training, improve the patient's HRQoL, and reduce the burden on the patient, family and society.

\section{External factors for the effect of intervention}

The most important and frequently mentioned factors are the internal motivation to exercise and the perceived influence of exercise, both of which have a positive effect on persistence; perceiving no effect will have a negative effect. Pain is an important factor affecting the transition from intrinsic motivation to exercise intention. Due to the involuntary nature of TheraBite ${ }^{\circledR}$ exercise, the key factors 
for developing internal motivation are the necessity of exercise, the setting of goals, the belief, self-discipline, and the fear of what might happen when not exercising.

Another external factor that influenced exercise adherence is the treatment of trismus by medical staff. Physicians not only act as motivators, but also indirectly affect compliance indirectly by reducing the fear of exercise. Some literature shows that family and lack of time has no significant impact on participants' exercise compliance.

Pain, anxiety, and medical workers have a positive and negative impact on the persistence of TheraBite ${ }^{\circledR}$ exercise. Initially, persistence is achieved by internal motivation (66). The second stage starts when the patient is aware of the impact of exercise, which is the most important factor in maintaining internal motivation.

\section{Conclusion and prospect}

Restricted mouth opening (trismus) in oral and maxillofacial malignant tumor has a profound influence on the quality of life of the patients, reducing the effects of rehabilitation and wellbeing. It is a significant complication of oral malignancy or its surgical and/or radiotherapy. As restriction becomes more severe and possible irreversible, the need for treatment becomes more urgent. Early treatment of restricted mouth opening (trismus) has the potential to prevent or minimise many of the consequences of this condition.

There are no specific treatments for trismus. The current strategy emphasizes prevention. Current strategies emphasize prevention, in instances of existing trismus, collaboration between professionals to establish pain control, prevent the progression of trismus, and restore function. Medical personnel can make a set of systematic, simple and feasible mouth-opening exercise methods to encourage patients to insist on long-term exercise to 1 year after radiotherapy, and give patients effective training guidance. Of course, how to develop a sophisticated, standardized and personalized TheraBite ${ }^{\circledR}$ rehabilitation systems for the prevention and treatment of restricted mouth opening or trismus, and to provide clinical guidance for the follow-up of patients with oral cancer treatment, remains to be studied.

\section{Acknowledgments}

Funding: Excellent Personnel Training Plan of Shanghai Health System (2018YQ34); Key Project of "Translational Medicine Crossover Research Fund" of Shanghai Jiao Tong
University (ZH20182DA15); Collaborative Innovation Team of Young Physicians in the Ninth People's Hospital Affiliated to Shanghai Jiao Tong University Medical College (QC201901).

\section{Footnote}

Conflicts of Interest: All authors have completed the ICMJE uniform disclosure form (available at https://fomm. amegroups.com/article/view/10.21037/fomm-20-52/ coif). CZ serves as an unpaid Associate Editor-in-Chief of Frontiers of Oral and Maxillofacial Medicine. All authors report that a patent open mouth trainer is pending.

Ethical Statement: The authors are accountable for all aspects of the work in ensuring that questions related to the accuracy or integrity of any part of the work are appropriately investigated and resolved.

Open Access Statement: This is an Open Access article distributed in accordance with the Creative Commons Attribution-NonCommercial-NoDerivs 4.0 International License (CC BY-NC-ND 4.0), which permits the noncommercial replication and distribution of the article with the strict proviso that no changes or edits are made and the original work is properly cited (including links to both the formal publication through the relevant DOI and the license). See: https://creativecommons.org/licenses/by-nc-nd/4.0/.

\section{References}

1. Siegel RL, Miller KD, Jemal A. Cancer statistics, 2019. CA Cancer J Clin 2019;69:7-34.

2. Ichimura $\mathrm{K}$, Tanaka T. Trismus in patients with malignant tumours in the head and neck. J Laryngol Otol 1993;107:1017-20.

3. Lee R, Slevin N, Musgrove B, et al. Prediction of posttreatment trismus in head and neck cancer patients. $\mathrm{Br} \mathrm{J}$ Oral Maxillofac Surg 2012;50:328-32.

4. Dijkstra PU, Kalk WW, Roodenburg JL. Trismus in head and neck oncology: a systematic review. Oral Oncol 2004;40:879-89.

5. Weber C, Dommerich S, Pau HW, et al. Limited mouth opening after primary therapy of head and neck cancer. Oral Maxillofac Surg 2010;14:169-73.

6. Louise Kent M, Brennan MT, Noll JL, et al. Radiationinduced trismus in head and neck cancer patients. Support Care Cancer 2008;16:305-9. 
7. Pauli N, Svensson U, Karlsson T, et al. Exercise intervention for the treatment of trismus in head and neck cancer - a prospective two-year follow-up study. Acta Oncol 2016;55:686-92.

8. Satheeshkumar PS, Mohan MP, Jacob J. Restricted mouth opening and trismus in oral oncology. Oral Surg Oral Med Oral Pathol Oral Radiol 2014;117:709-15.

9. Rapidis AD, Dijkstra PU, Roodenburg JL, et al. Trismus in patients with head and neck cancer: etiopathogenesis, diagnosis and management. Clin Otolaryngol 2015;40:516-26.

10. Shulman DH, Shipman B, Willis FB. Treating trismus with dynamic splinting: a cohort, case series. Adv Ther 2008;25:9-16.

11. Lee YC, Wong TY, Shieh SJ, et al. Trismus release in oral cancer patients. Ann Plast Surg 2012;69:598-601.

12. LENT SOMA tables. Radiother Oncol 1995;35:17-60.

13. Johnson J, van As-Brooks CJ, Fagerberg-Mohlin B, et al. Trismus in head and neck cancer patients in Sweden: incidence and risk factors. Med Sci Monit 2010;16:CR278-82.

14. Watters AL, Cope S, Keller MN, et al. Prevalence of trismus in patients with head and neck cancer: A systematic review with meta-analysis. Head Neck 2019;41:3408-21.

15. Oh HK, Chambers MS, Martin JW, et al. Osteoradionecrosis of the mandible: treatment outcomes and factors influencing the progress of osteoradionecrosis. J Oral Maxillofac Surg 2009;67:1378-86.

16. Ogawa K, Toita T, Kakinohana Y, et al. Postoperative radiotherapy for squamous cell carcinoma of the maxillary sinus: analysis of local control and late complications. Oncol Rep 2001;8:315-9.

17. Cohen EG, Deschler DG, Walsh K, et al. Early use of a mechanical stretching device to improve mandibular mobility after composite resection: a pilot study. Arch Phys Med Rehabil 2005;86:1416-9.

18. 18. Scott B, D'Souza J, Perinparajah N, et al. Longitudinal evaluation of restricted mouth opening (trismus) in patients following primary surgery for oral and oropharyngeal squamous cell carcinoma. Br J Oral Maxillofac Surg 2011;49:106-11.

19. Sciubba JJ, Goldenberg D. Oral complications of radiotherapy. Lancet Oncol 2006;7:175-83.

20. Stubblefield MD, Manfield L, Riedel ER. A preliminary report on the efficacy of a dynamic jaw opening device (dynasplint trismus system) as part of the multimodal treatment of trismus in patients with head and neck cancer. Arch Phys Med Rehabil 2010;91:1278-82.
21. Miller FR, Wanamaker JR, Lavertu P, et al. Magnetic resonance imaging and the management of parapharyngeal space tumors. Head Neck 1996;18:67-77.

22. Jiang GL, Ang KK, Peters LJ, et al. Maxillary sinus carcinomas: natural history and results of postoperative radiotherapy. Radiother Oncol 1991;21:193-200.

23. Nishino H, Miyata M, Morita M, et al. Combined therapy with conservative surgery, radiotherapy, and regional chemotherapy for maxillary sinus carcinoma. Cancer 2000;89:1925-32.

24. Ozsaran Z, Yalman D, Baltalarli B, et al. Radiotherapy in maxillary sinus carcinomas: evaluation of 79 cases. Rhinology 2003;41:44-8.

25. Karakoyun-Celik O, Norris CM, Jr., Tishler R, et al. Definitive radiotherapy with interstitial implant boost for squamous cell carcinoma of the tongue base. Head Neck 2005;27:353-61.

26. Eisen MD, Weinstein GS, Chalian A, et al. Morbidity after midline mandibulotomy and radiation therapy. Am J Otolaryngol 2000;21:312-7.

27. Bertrand J, Luc B, Philippe M, et al. Anterior mandibular osteotomy for tumor extirpation: a critical evaluation. Head Neck 2000;22:323-7.

28. Yeh SA, Tang Y, Lui CC, et al. Treatment outcomes and late complications of 849 patients with nasopharyngeal carcinoma treated with radiotherapy alone. Int J Radiat Oncol Biol Phys 2005;62:672-9.

29. Fuchs S, Rodel C, Brunner T, et al. Patterns of failure following radiation with and without chemotherapy in patients with nasopharyngeal carcinoma. Onkologie 2003;26:12-8.

30. Wolden SL, Zelefsky MJ, Hunt MA, et al. Failure of a $3 \mathrm{D}$ conformal boost to improve radiotherapy for nasopharyngeal carcinoma. Int J Radiat Oncol Biol Phys 2001;49:1229-34.

31. van der Geer SJ, van Rijn PV, Kamstra JI, et al. Criterion for trismus in head and neck cancer patients: a verification study. Support Care Cancer 2019;27:1129-37.

32. Nguyen TD, Panis X, Froissart D, et al. Analysis of late complications after rapid hyperfractionated radiotherapy in advanced head and neck cancers. Int J Radiat Oncol Biol Phys 1988;14:23-5.

33. Goldstein M, Maxymiw WG, Cummings BJ, et al. The effects of antitumor irradiation on mandibular opening and mobility: a prospective study of 58 patients. Oral Surg Oral Med Oral Pathol Oral Radiol Endod 1999;88:365-73.

34. Wang CJ, Huang EY, Hsu HC, et al. The degree and time-course assessment of radiation-induced trismus 
occurring after radiotherapy for nasopharyngeal cancer. Laryngoscope 2005;115:1458-60.

35. Bensadoun RJ, Riesenbeck D, Lockhart PB, et al. A systematic review of trismus induced by cancer therapies in head and neck cancer patients. Support Care Cancer 2010;18:1033-8.

36. O'Sullivan B, Levin W. Late radiation-related fibrosis: pathogenesis, manifestations, and current management. Semin Radiat Oncol 2003;13:274-89.

37. Scott B, Butterworth C, Lowe D, et al. Factors associated with restricted mouth opening and its relationship to health-related quality of life in patients attending a Maxillofacial Oncology clinic. Oral Oncol 2008;44:430-8.

38. Teguh DN, Levendag PC, Voet P, et al. Trismus in patients with oropharyngeal cancer: relationship with dose in structures of mastication apparatus. Head Neck 2008;30:622-30.

39. Chen YY, Zhao C, Wang J, et al. Intensity-modulated radiation therapy reduces radiation-induced trismus in patients with nasopharyngeal carcinoma: a prospective study with $>5$ years of follow-up. Cancer 2011;117:2910-6.

40. Tveterås K, Kristensen S. The aetiology and pathogenesis of trismus. Clin Otolaryngol Allied Sci 1986;11:383-7.

41. Eipe $\mathrm{N}$. The chewing of betel quid and oral submucous fibrosis and anesthesia. Anesth Analg 2005;100:1210-3.

42. Johnson NW, Warnakulasuriya S, Gupta PC, et al. Global oral health inequalities in incidence and outcomes for oral cancer: causes and solutions. Adv Dent Res 2011;23:237-46.

43. Bengtson BP, Schusterman MA, Baldwin BJ, et al. Influence of prior radiotherapy on the development of postoperative complications and success of free tissue transfers in head and neck cancer reconstruction. Am J Surg 1993;166:326-30.

44. Bhatia KS, King AD, Paunipagar BK, et al. MRI findings in patients with severe trismus following radiotherapy for nasopharyngeal carcinoma. Eur Radiol 2009;19:2586-93.

45. Lindblom U, Garskog O, Kjellen E, et al. Radiationinduced trismus in the ARTSCAN head and neck trial. Acta Oncol 2014;53:620-7.

46. Wranicz P, Herlofson BB, Evensen JF, et al. Prevention and treatment of trismus in head and neck cancer: A case report and a systematic review of the literature. Scand J Pain 2010;1:84-8.

47. Vissink A, Jansma J, Spijkervet FK, et al. Oral sequelae of head and neck radiotherapy. Crit Rev Oral Biol Med 2003;14:199-212.

48. Ling IS, Larsson B. Individualized pharmacological treatment of oral mucositis pain in patients with head and neck cancer receiving radiotherapy. Support Care Cancer 2011;19:1343-50.

49. Scherpenhuizen A, van Waes AM, Janssen LM, et al. The effect of exercise therapy in head and neck cancer patients in the treatment of radiotherapy-induced trismus: A systematic review. Oral Oncol 2015;51:745-50.

50. Chua DT, Lo C, Yuen J, et al. A pilot study of pentoxifylline in the treatment of radiation-induced trismus. Am J Clin Oncol 2001;24:366-9.

51. King GE, Scheetz J, Jacob RF, et al. Electrotherapy and hyperbaric oxygen: promising treatments for postradiation complications. J Prosthet Dent 1989;62:331-4.

52. Stubblefield MD, Levine A, Custodio CM, et al. The role of botulinum toxin type $\mathrm{A}$ in the radiation fibrosis syndrome: a preliminary report. Arch Phys Med Rehabil 2008;89:417-21.

53. Bhrany AD, Izzard M, Wood AJ, et al. Coronoidectomy for the treatment of trismus in head and neck cancer patients. Laryngoscope 2007;117:1952-6.

54. Huang IY, Wu CF, Shen YS, et al. Importance of patient's cooperation in surgical treatment for oral submucous fibrosis. J Oral Maxillofac Surg 2008;66:699-703.

55. Mehrotra D, Pradhan R, Gupta S. Retrospective comparison of surgical treatment modalities in 100 patients with oral submucous fibrosis. Oral Surg Oral Med Oral Pathol Oral Radiol Endod 2009;107:e1-10.

56. Chan RC, Wei FC, Tsao CK, et al. Free flap reconstruction after surgical release of oral submucous fibrosis: long-term maintenance and its clinical implications. J Plast Reconstr Aesthet Surg 2014;67:344-9.

57. Mardini S, Chang YM, Tsai CY, et al. Release and free flap reconstruction for trismus that develops after previous intraoral reconstruction. Plast Reconstr Surg 2006;118:102-7.

58. Lin PY, Chen CC, Kuo YR, et al. Simultaneous reconstruction of head and neck defects following tumor resection and trismus release with a single anterolateral thigh donor site utilizing a lateral approach to flap harvest. Microsurgery 2012;32:289-95.

59. National Institutes of Health Consensus Development Conference on Oral Complications of Cancer Therapies: Diagnosis, Prevention, and Treatment. Bethesda, Maryland, April 17-19, 1989. NCI Monogr 1990:1-184.

60. Graff P, Lapeyre M, Desandes E, et al. Impact of intensitymodulated radiotherapy on health-related quality of life for head and neck cancer patients: matched-pair comparison with conventional radiotherapy. Int J Radiat Oncol Biol 
Phys 2007;67:1309-17.

61. Epstein JB, Thariat J, Bensadoun RJ, et al. Oral complications of cancer and cancer therapy: from cancer treatment to survivorship. CA Cancer J Clin 2012;62:400-22.

62. Li YH, Chang WC, Chiang TE, et al. Mouth-opening device as a treatment modality in trismus patients with head and neck cancer and oral submucous fibrosis: a prospective study. Clin Oral Investig 2019;23:469-76.

63. Kamstra JI, Roodenburg JL, Beurskens CH, et al. TheraBite exercises to treat trismus secondary to head and neck cancer. Support Care Cancer 2013;21:951-7.

doi: $10.21037 /$ fomm-20-52

Cite this article as: $\mathrm{Wu} \mathrm{H}$, Zhou Z, Zhang C, Shen S, Liu J, Zhang C. The progress of post-treatment restricted mouth opening in oral and maxillofacial malignant tumor patients. Front Oral Maxillofac Med 2021;3:7.
64. Lee R, Molassiotis A, Rogers SN, et al. Protocol for the trismus trial-therabite versus wooden spatula in the amelioration of trismus in patients with head and neck cancer: randomised pilot study. BMJ Open 2018;8:e021938.

65. Dijkstra PU, Huisman PM, Roodenburg JL. Criteria for trismus in head and neck oncology. Int J Oral Maxillofac Surg 2006;35:337-42.

66. Melchers LJ, Van Weert E, Beurskens CH, et al. Exercise adherence in patients with trismus due to head and neck oncology: a qualitative study into the use of the Therabite. Int J Oral Maxillofac Surg 2009;38:947-54. 